\title{
Lentiviral-mediated overexpression of long non-coding RNA GAS5 reduces invasion by mediating MMP2 expression and activity in human melanoma cells
}

\author{
LONG CHEN $^{1 *}$, HUIXIN YANG $^{1 *}$, YANBIN XIAO $^{2 *}$, XIAOXIA TANG $^{3}$, YUQIAN LI $^{1}$, \\ QIAOQIAO HAN ${ }^{1}$, JUNPING FU ${ }^{2}$, YUYE YANG ${ }^{1}$ and YUECHUN ZHU ${ }^{1}$ \\ ${ }^{1}$ Department of Biochemistry and Molecular Biology of Kunming Medical University; \\ ${ }^{2}$ Department of Orthopaedic Surgery, The Third Affiliated Hospital of Kunming Medical University; \\ ${ }^{3}$ Department of Pharmacy, The Second Affiliated Hospital of Kunming Medical University, Yunnan, P.R. China
}

Received December 1, 2015; Accepted January 7, 2016

DOI: 10.3892/ijo.2016.3377

\begin{abstract}
The present study evaluated the effects of long non-coding RNA GAS5 on the migration and invasion of melanoma cells. Using the SK-Mel-110 melanoma cell line, we stably expressed GAS5, visualized the distribution of GAS5 by RNA fluorescence in situ hybridization (FISH) and examined changes in cell migration and invasion with Transwell assays. In GAS5 overexpressed SK-Mel-110 cells, migrated and invaded cells decreased by 65.3 and $55.6 \%$, respectively. Moreover, the MMP2 protein level, and its activity was downregulated by 67.9 and $15.8 \%$, respectively. Overexpressing lncRNA GAS5 inhibited the migration and invasion ability of melanoma SK-Mel-110 cells, partially by decreasing the MMP2 expression and its activity. This study is the first to reveal a potential relationship between IncRNA GAS5 and the migration and invasion of melanoma.
\end{abstract}

\section{Introduction}

Melanoma is an aggressive disease with an ever-increasing incidence rate and is the deadliest known form of skin cancer. In 2015, an estimated 80,100 new cases and 13,340 deaths from skin cancer are expected in the United States, with melanoma accounting for $75 \%$ of all skin cancer deaths (1). Although targeted therapies and immunotherapies have revolutionized melanoma treatment and improved the quality of life of advanced melanoma patients, few drugs are available

Correspondence to: Dr Yuechun Zhu, Department of Biochemistry and Molecular Biology of Kunming Medical University, Yunnan, P.R. China

E-mail: zhuyuechun20091119@163.com

${ }^{*}$ Contributed equally

Abbreviations: GAS5, growth arrest specific transcript 5

Key words: long non-coding RNA, growth arrest specific transcript 5, malignant melanoma, invasion (only seven as of 2014), and drug resistance is increasing (2). Therefore, further study of the progression of metastatic melanoma and the innovation of new, targeted therapies are desperately needed.

Long non-coding RNAs (lncRNAs) are RNA molecules with lengths of more than 200 nucleotides and little probability of translation into a protein (3). Considered useless and subsequently ignored for several decades, lncRNA is now attracting more and more attention, and increasing evidence has suggested that lncRNAs participate in universal biological processes such as cell differentiation, proliferation, migration and invasion (4). Studies have also revealed that some lncRNAs, such as HOTAIR and growth arrest specific transcript 5 (GAS5), can function as tumor promoters or suppressors, respectively $(5,6)$. Other lncRNAs function by providing a scaffold through which proteins can adhere, accumulate and exert their functions, suggesting that specific RNA secondary structures are needed for lncRNAs to carry out their functions $(7,8)$. Located at 1q25 and with a mature length of 650 bases, the lncRNA growth arrest-specific transcript 5 (GAS5) comprises 12 exons and encodes 10 box C/D snoRNAs within its introns (9). GAS5 was originally isolated from NIH 3T3 cells by Schneider et al with the purpose of identifying potential tumor suppressor genes enriched during growth arrest induced by serum starvation (10). GAS5 transcript levels are significantly reduced in a spectrum of cancers including breast, prostate, glioblastoma, renal clear cell, bladder, hepatocellular, pancreatic, non-small cell lung, colorectal, cervical and multiple myeloma (11-21). Importantly, aberrant GAS5 expression was significantly correlated with metastasis in cervical cancer (21) and hepatocellular carcinoma (15).

However, despite the past research, the expression profile and biological role of GAS5 and its mechanism in melanoma metastasis remain largely unknown. The tumorigenesis process and progression of melanoma to metastasis is generally recognized as a multistep process in which the cellular and molecular mechanisms change. The first step of invasion, which is the degradation of the extracellular matrix, is regulated by the matrix metalloproteinase (MMP) family, which plays a key role in tumor cell invasion and metastasis 
(22). MMP2, which was shown to be specifically involved in type IV collagen degradation, is also secreted by a wide range of melanoma cells (23). Specifically, MMP2 is detected in the early stages of melanocytic tumor progression (24). Although MMP13 is also gelatinolytic and type IV collagenolytic, it is prone to degrading type I, II and III collagens (25). Previous studies have shown that upregulated MMP13 and its activity in melanoma cell lines are involved in the metastasis of melanoma $(26,27)$. A recent study revealed that MMP13 not only cleaves laminin-5 into small fragments to accelerate tumor metastasis but also disrupts vasculogenic mimicry in the presence of cleaved laminin-5, exerting dual effects on melanoma (28).

The SK-Mel-110 melanoma cell line, isolated from a primary melanoma patient, has been cultured in vitro for nearly 30 years and has been divided many times, which is indicative of an immortal cell line (29). SK-Mel-110 cells grow actively, with a doubling time of $\sim 20 \mathrm{~h}$, and are tumorigenic in immunodeficient nude mice (30). They have been used widely at several melanoma research centers for the study of melanogenesis, which investigates the role that autophagy and apoptotic resistance play in the pathogenesis of melanoma, and in various pharmacological and toxicological studies (31-33). In the present study, substantial downregulation of GAS5 was detected in SK-Mel-110 cells, and therefore it was selected for further study.

Based on these reports, we hypothesized that GAS5 may function as a tumor suppressor by inhibiting melanoma cell invasiveness via the regulation of MMP2 or MMP13. To further understand the role of GAS5 in the metastasis of melanoma, we overexpressed GAS5 in SK-Mel-110 cells. Using this model, the effects of GAS5 on melanoma migration and invasion, and the potential correlation between GAS5 and MMPs, were investigated.

\section{Materials and methods}

Cell culture. The human melanoma cell line A375 was purchased from ATCC (Manassas, VA, USA) and Hacat, SK-Mel-110, SK-Mel-28 and M21 were purchased from the Shanghai Institutes for Biological Science (SIBS), China Academy of Science (CAS). Cells were cultured in Dulbecco's modified Eagle's medium (DMEM, Invitrogen, Carlsbad, CA, USA) supplemented with $5 \%$ fetal bovine serum (FBS, Life Technologies, Burlington, Ontario, Canada), $100 \mathrm{U} / \mathrm{ml}$ penicillin and $100 \mu \mathrm{g} / \mathrm{ml}$ streptomycin at $37^{\circ} \mathrm{C}$ in $5 \%$ (v/v) $\mathrm{CO}_{2}$. Cells were maintained without exceeding $0.5 \times 10^{6}$ cells $/ \mathrm{ml}$. 293T human embryonic kidney (HEK) cells were obtained from the Cell Bank at the Chinese Academy of Science and cultured in DMEM containing 10\% (v/v) FBS and $100 \mu \mathrm{g} / \mathrm{ml}$ streptomycin at $37^{\circ} \mathrm{C}$ in $5 \%(\mathrm{v} / \mathrm{v}) \mathrm{CO}_{2}$.

Predicting secondary structure of GAS5. Secondary structural prediction was performed using the RNA Vienna Package on the webserver (http://rna.tbi.univie.ac.at/) (34,35). This program predicts RNA secondary structure based on minimum energy requirements and pair probabilities.

Total RNA extraction and detection of GAS5 by qRT-PCR. Total RNA was isolated from the transfected cells using TRIzol reagent (Invitrogen) according to the manufacturer's protocol. Briefly, after removing the culture medium, TRIzol reagent was added to each well, followed by incubation at room temperature for $10 \mathrm{~min}$ and centrifugation at $12,000 \mathrm{~g}$ for $10 \mathrm{~min}$. The supernatant was removed to obtain the precipitated RNA. After washing with $75 \%$ ethanol, the dried RNA pellet was then resuspended in RNase-free water and stored at $-80^{\circ} \mathrm{C}$ until use. cDNA was synthesized using Oligo(dT) and M-MLV reverse transcriptase (M1701, Promega). GAS5 primers (F, 5'-GCACACAGGCATTAGAC AGA-3'; R, 5'-AAGCCGACTCTCCATACCTT-3'), and the U6 primers (F, 5'-CTCGCTTCGGCAGCACA-3'; R, 5'-AACG CTTCACGAATTTGCGT-3') were synthesized by Shengon (Shanghai, China). Real-time PCR was performed using a sequence detection system (ABI 7300; Applied Biosystems, Foster City, CA, USA). The $20-\mu 1$ reaction mixture contained $10 \mu \mathrm{l}$ of SYBR green PCR master mix (A6020, Promega), $1 \mu 1$ of cDNA template, $1 \mu \mathrm{l}$ of PCR forward primer, $1 \mu \mathrm{l}$ of PCR reverse primer and $7 \mu \mathrm{l}$ of RNA-free water. PCR running conditions were as follows: $15 \mathrm{sec}$ at $95^{\circ} \mathrm{C}$ for the initial denaturation, 30 cycles of $5 \mathrm{sec}$ at $95^{\circ} \mathrm{C}$ and $30 \mathrm{sec}$ at $60^{\circ} \mathrm{C}$ and $1 \mathrm{~min}$ at $95^{\circ} \mathrm{C}$ for annealing. The threshold cycle (Ct value), which is the cycle number at which the amount of amplified gene of interest reaches a fixed threshold, was subsequently determined. GAS5 gene expression was compared to control cells by qRT-PCR using the $2-\Delta \Delta \mathrm{Ct}$ method with U6 as an internal control. The results are expressed as the mean $\pm \mathrm{SD}$ from three independent experiments.

Constructing a recombinant vector containing the GAS5 gene. The mature human GAS5 gene (NM_002578.2) was synthesized by Genewiz (Suzhou, China). The primers are as follows: GAS5-XhoI-F, 5'-ccgetcgagTTTCGAGGTAGGA GTCGACTCCTGTG-3'; and GAS5-BamHI-R, 5'-cgcggatcc TTTTTTTTTTTTTTTTTTTGGATTGCAAA-3'. These primers contain both XhoI and BamHI restriction sites (underlined). The $25-\mu 1$ reaction mixture contained $2.5 \mu l$ of $2 \mathrm{mM}$ dNTP mixture, $2.5 \mu \mathrm{l}$ of 10X KOD buffer, $1.5 \mu 1$ of $25 \mathrm{mM}$ $\mathrm{MgSO}_{4}, 0.5 \mu \mathrm{l}$ of synthesized template, $0.3 \mu \mathrm{l}$ of PCR forward primer, $0.3 \mu \mathrm{l}$ of PCR reverse primer, $0.3 \mu \mathrm{l}$ of KOD Plus Neo and $17.1 \mu \mathrm{l}$ of RNA-free water. PCR running conditions were as follows: $3 \mathrm{~min}$ at $94^{\circ} \mathrm{C}$ for the initial denaturation, 30 cycles of $15 \mathrm{sec}$ at $98^{\circ} \mathrm{C}$ and $15 \mathrm{sec}$ at $58^{\circ} \mathrm{C}$ and $30 \mathrm{sec}$ at $95^{\circ} \mathrm{C}$ for annealing. The resulting product was kept at $16^{\circ} \mathrm{C}$ until further use. After isolation and purification of the PCR product, the GAS5 gene was inserted into the corresponding site of the linearized expression vector pLVX-IRES-ZsGreen1 (data not shown), downstream of the cytomegalovirus (CMV) promoter. The recombinant plasmid was then transformed into DH5 $\alpha$ competent cells $(36,37)$ (Fig. 1).

Identifying positive recombinant vectors, packaging lentivirus and determining lentivirus titers. Positive recombinant clones were examined and verified by DNA sequencing (data not shown). To generate lentiviruses, three individual lentiviral vectors were required: the pLVX-IRES-ZsGreen1GAS5 vector, the pHelper 1.0 vector (gag/pol element) and the pHelper 2.0 vector (VSVG element). Lentiviruses were generated in HEK293T cells as described previously, with minor modifications (38). In brief, $20 \mu \mathrm{g}$ of pLVX-IRES-ZsGreen1GAS5 vector, $10 \mu \mathrm{g}$ of pHelper 1.0 and $10 \mu \mathrm{g}$ of pHelper 2.0 were 
cotransfected into $293 \mathrm{~T}$ cells with the help of a transfection reagent (Lipofectamine 2000, Invitrogen). Seventy-two hours after transfection, supernatants were removed and filtered (0.45- $\mu \mathrm{m}$ filter, Millipore). Virus was collected by ultracentrifugation for $60 \mathrm{~min}$ at $20,000 \mathrm{rpm}\left(4^{\circ} \mathrm{C}\right)$ and stored at $-80^{\circ} \mathrm{C}$ until further use. The virus-containing supernatant was titrated onto SK-Mel-110 cells to determine the titers needed to transduce $95 \%$ of the cells ( $6 \times 10^{6}$ transduction units $\left./ \mathrm{ml}\right)$. Mock viruses were generated by the same procedure using otherwise identical vectors lacking GAS5 cDNA.

The GAS5 gene was observed as a band between 500-750 bp, which correlated with the expected size of $651 \mathrm{bp}$ (data not shown). DNA sequencing confirmed that the tested sequence was identical to that in GenBank/NCBI (data not shown).

Establishing stable GAS5 overexpression in human melanoma cells. For transduction, SK-Mel-100 cells were inoculated in a 24 -well plate and cultured until they reached $50-60 \%$ confluence. The experimental virus solution, pLVX-IRESZsGreen1-GAS5 (GAS5 overexpression group), and the control virus solution, ZsGreen 1 (control group), were thawed on ice and added to the culture at a multiplicity of infection (MOI) of 5 for $72 \mathrm{~h}$ in the presence of polybrene (Santa Cruz Biotech). Infection was carried out in humidified air containing $5 \% \mathrm{CO}_{2}$ at $37^{\circ} \mathrm{C}$. ZsGreen1 expression was visualized using a fluorescence microscope. The fluorescent signal indicating ZsGreen1 expression in the stably-transfected cells was observed after repeated freeze-thaw cycles to determine the stability of the transfected genes, after which the experimental procedures were performed.

RNA fluorescence in situ hybridization. Oligonucleotide modified probes for human IncRNA GAS5 (5'-TAMRACAGGAGCAGAACCATTAAGCTGGTCCAGGCAAGT-3'-

TAMRA) and a negative/scramble control were used for RNA fluorescence in situ hybridization (FISH). Procedures were carried out based on a previous study (39), with slight modification. Briefly, the cell suspension was pipetted onto autoclaved glass slides. The following day, the slides were washed in PBS and fixed in $4 \%$ paraformaldehyde. To permeabilize the cell membrane, $0.2 \mathrm{M} \mathrm{HCl}$ was used. The slides were then treated with the protease reagent; this activity was stopped by the addition of glycine. After dehydration with 70,85 and $100 \%$ ethanol, hybridization was performed at $37^{\circ} \mathrm{C}$ overnight in a dark moist chamber. After hybridization, slides were washed three times in $50 \%$ formamide/2X SSC for $5 \mathrm{~min}$ each at room temperature. These slides were counterstained with DAPI. The images were acquired using a confocal microscope.

Cell mobility and invasion using a Transwell assay. Cell mobility was examined using a 24-well chamber plate containing a polycarbonate membrane with an $8-\mu \mathrm{m}$ pore size. DMEM with $10 \%$ FBS $(0.8 \mathrm{ml})$ served as a chemoattractant in the lower chamber. First, $1 \times 10^{4}$ cells in $0.2 \mathrm{ml}$ of serum-free medium were added to the inserts and incubated for $24 \mathrm{~h}$ at $37^{\circ} \mathrm{C}$ and $5 \% \mathrm{CO}_{2}$. Cells remaining on the upper membrane surface of the inserts were then removed with a cotton swab, and the total number of cells that migrated into the lower chamber was counted. A cell invasion assay was performed in a similar manner to the cell motility assay with some modifications. Briefly, $5 \times 10^{4}$ cells in serum-free DMEM were added to the upper chambers of transwells with $8-\mu \mathrm{m}$ pore size polycarbonate membranes and precoated with a thin layer of Matrigel. DMEM with 10\% FBS was used as a chemoattractant in the lower chamber. The total numbers of cells in the lower chamber were counted after $36 \mathrm{~h}$ of incubation at $37^{\circ} \mathrm{C}$ with $5 \% \mathrm{CO}_{2}$.

Western blotting to demonstrate MMP2 and MMP13 protein expression. For western blots, cell lysates and culture supernatants were prepared using RIPA buffer $(50 \mathrm{mM}$ Tris- $\mathrm{HCl}$, $\mathrm{pH}$ 7.4) with $1 \mathrm{mM}$ PMSF and incubated at $4^{\circ} \mathrm{C}$ for $30 \mathrm{~min}$. The lysates were centrifuged at $12,000 \mathrm{rpm}$ for $10 \mathrm{~min}$ at $4^{\circ} \mathrm{C}$. Protein concentrations were determined using a NanoDrop ND-1000 spectrophotometer (Thermo Scientific). Samples (20 $\mu \mathrm{g}$ of protein) were separated by SDS-PAGE using $5 \%$ stacking and $12 \%$ separating gels and subsequently transferred onto a PVDF membrane (Millipore). After blocking in Tris-buffer (50 mM Tris, pH 7.5) containing 5\% skim milk, the membrane was incubated overnight at $4^{\circ} \mathrm{C}$ with antibodies to detect MMP2 (ab110186, 1:2000; Abcam), MMP13 (ab39012, 1:1000; Abcam) and GAPDH (Ab8227, 1:1000; Abcam), followed by incubation for $1 \mathrm{~h}$ at room temperature with a corresponding HRP-conjugated goat anti-rabbit IgG secondary antibody (sc-2004, 1:1000; Santa Cruz). Protein expression was visualized after extensive washing using an enhanced chemiluminescence advanced detection kit (34077, Thermo Scientific) and quantified with analysis software (Quantity One; Bio-Rad Laboratories Inc., Hercules, CA, USA).

MMP2 activity assay. MMP2 activity in cell extracts and culture supernatants was detected using an MMP2 activity assay kit (GMS50070.1, Genmed, Shanghai, China) according to the manufacturer's instructions. For this assay, MMP2 activity was detected by the fluorescence resonance energy transfer method (FRET), which involves the transfer of excited energy from a donor (with a short wavelength) to an acceptor (with a long wavelength) so that the emitted photon from the donor, whose reaction is dependent on proximity to the acceptor, is quenched. The donor fluorescent probe, 7-methoxycoumarin, labeled with the peptide substrate PLGLAR, was quenched by the acceptor using dinitrobenzene diamino isobutyryloxy. With the hydrolysis of the polypeptide Gly-Leu bond by MMP2, the strongly fluorescent 7-methoxycoumarin polypeptide fragment was released. Based on this fluorescence intensity (excited at $330 \mathrm{~nm}$ and emitted at $400 \mathrm{~nm}$ ) and standard curve, we obtained the 7-methoxycoumarin concentration. The specific activity of MMP2 was then calculated using the following formula: MMP2 activity $(\mathrm{nmol} / \mathrm{mg} / \mathrm{min})=$ 7-methoxycoumarin concentration $(\mu \mathrm{mol} / \mathrm{l}) \mathrm{x}$ dilution times $\div$ $5 \mathrm{~min} \div$ the concentration of protein sample $(\mathrm{mg} / \mathrm{ml})$.

Statistical analysis. Data are expressed as the mean \pm standard deviation of triplicate samples. Statistical analysis was performed using Student's t-test. Differences were considered to be statistically significant at $\mathrm{P}<0.05$ (two-tailed). All statistical analysis was performed with GraphPad Prism version 6.0e for Mac OS X (GraphPad Software, La Jolla, CA, USA). 
A

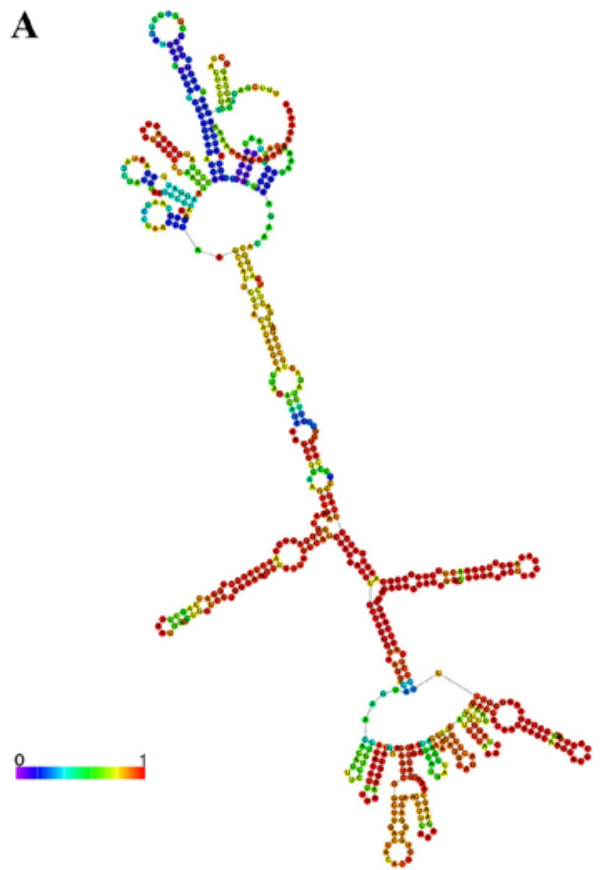

B

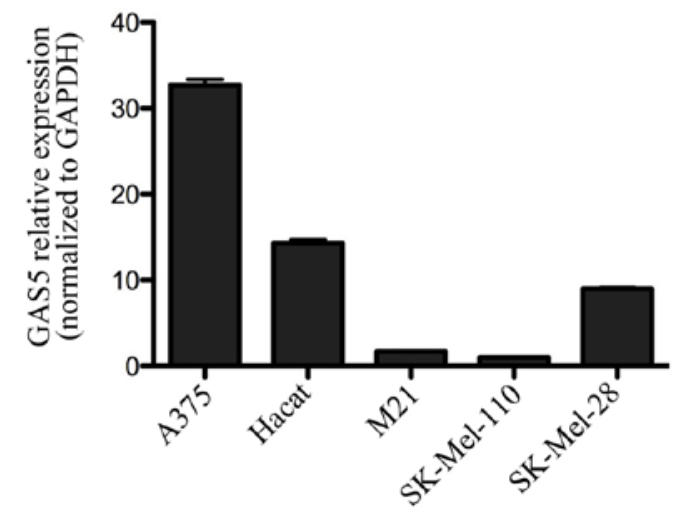

Figure 1. GAS5 contains a complicated secondary structure and its expression level varies in different melanoma cell lines. (A) The optimal secondary structure with a minimum free energy of $-193.80 \mathrm{kcal} / \mathrm{mol}$. The secondary structure of GAS5 was predicted by the RNAfold webserver (http://rna.tbi.univie. ac.at/cgi-bin/RNAfold.cgi). The structure is colored according to base-pairing probabilities. For unpaired regions, the color denotes the probability of being unpaired. (B) GAS5 expression levels vary greatly among five different melanoma cell lines and are sharply downregulated in SK-Mel-110 cells. Data are presented as the mean $\pm \mathrm{SD}$ from three independent experiments.

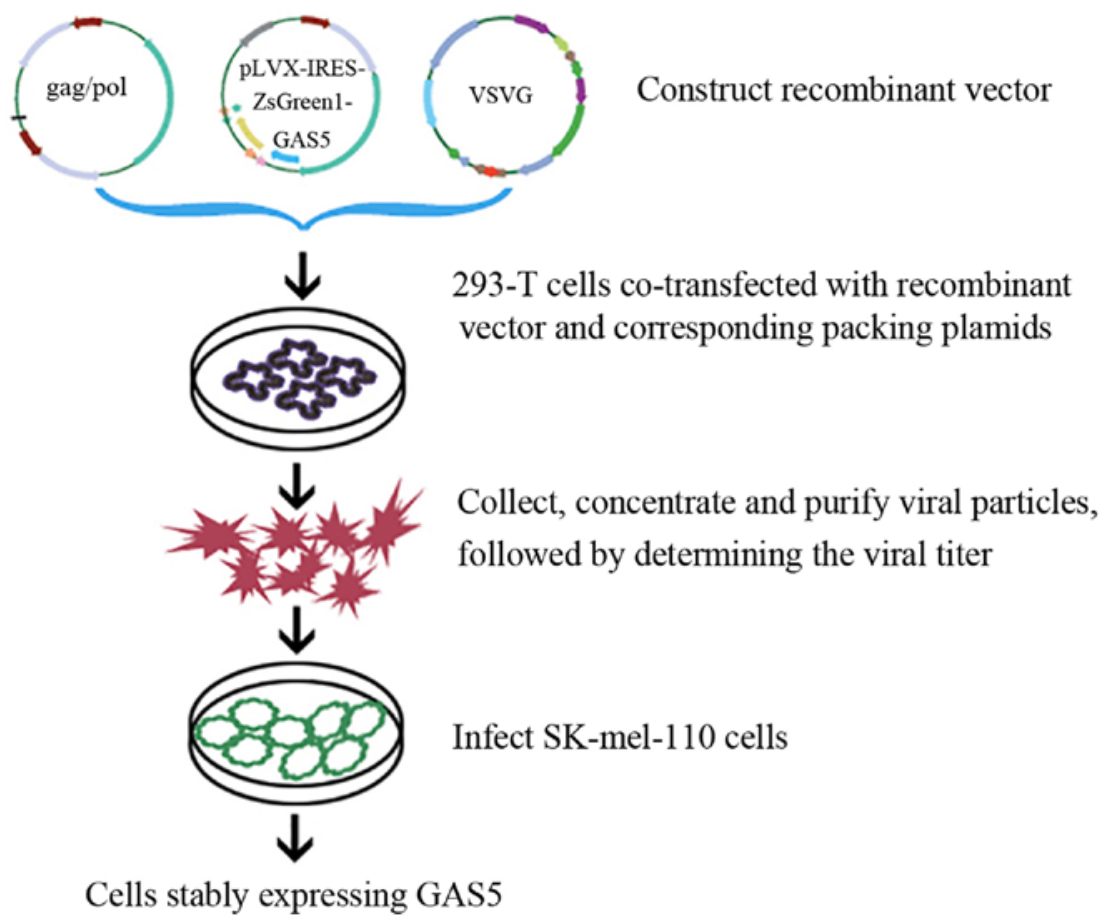

Figure 2. Schematic illustration of the establishment of a GAS5 stably expressing lentivirus-based vector. The GAS5 sequence (NR_002578.2) was cloned into a shuttle vector. The recombinant lentiviral vector was packaged into $293 \mathrm{~T}$ cells along with plasmids encoding Gag/Pol and VSVG. The recombinant lentiviral particles were collected, amplified, concentrated and suitably titrated for the transduction of SK-Mel-110 cells.

\section{Results}

The secondary structure of GAS5 and its mRNA levels in the SK-Mel-110 cell line. We first predicted the secondary structure of GAS5 using RNAfold and found that it contains a complex stem-loop structure, indicating that GAS5 may perform a complex and important biological function (Fig. 2A). Next, we examined lncRNA GAS5 expression levels in HaCaT, 

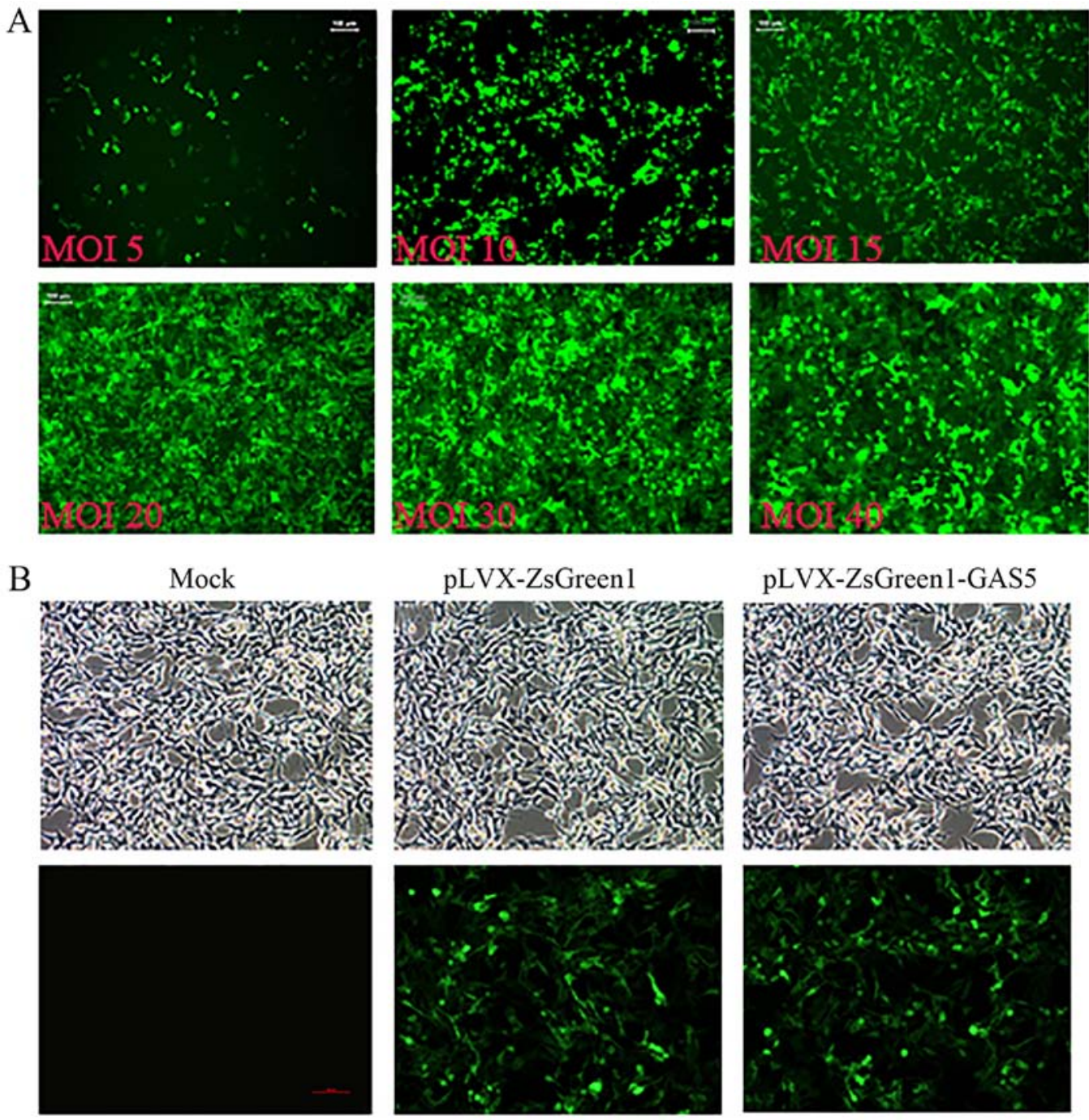

Figure 3. Infection efficiency of human SK-Mel-110 cells determined by examining ZsGreen1. (A) As the number of viral particles increased, the number of SK-Mel-110 cells expressing green fluorescent protein also increased. However, there was no significant increase observed with a MOI >20; accordingly, an MOI of 20 was selected for further experiments. (B) Green fluorescent protein expression was observed by fluorescence microscopy $72 \mathrm{~h}$ after transfection. The upper images were taken under normal light and the lower images under fluorescence. Mock, negative control group without transfected cells; pLVXZsGreen1, negative GFP control group with cells transfected with ZsGreen1 lentiviral vector alone; pLVX-ZsGreen1-GAS5, experimental group (GAS5 overexpression group) with cells transfected with GAS5-ZsGreen1 lentiviral vectors. N, normal light; F, fluorescent field. Scale bars, $50 \mu \mathrm{m}$.

A375, SK-Mel-28, SK-Mel-110 and M21 cells by qRT-PCR. After normalizing to GAPDH, the GAS5 expression level in SK-Mel-110 cells was significantly lower than that detected in other melanoma cell lines (Fig. 2B).

Transfection efficiency and selection of a stably expressing GAS5 cell line. To investigate GAS5 function in SK-Mel-110 cell lines, we overexpressed this gene by infecting SK-Mel-110 cells with pLVX-IRES-ZsGreen1-GAS5-containing lentivirus. The GAS5 fragment was amplified by reverse transcription polymerase chain reaction (RT-PCR) and verified by DNA sequencing. The use of different MOIs was designed to verify the most efficient dose of virus. After infection, green fluorescence in the cytoplasm of SK-Mel-110 cells was observed by a fluorescence microscope, and the efficiency of the infection, or the fraction of SK-Mel-110 cells with ZsGreen1 fluorescence, was quantified. As the number of viral particles increased, the number of SK-Mel-110 cells with ZsGreen1 fluorescence also increased. When the MOI reached $>40$, the number of green fluorescent SK-Mel-110 cells did not increase significantly (Fig. 3A). Consequently, a MOI of 40 was chosen for subsequent experiments. The transfection efficiency was calculated as the ratio of the number of fluorescence-positive cells to the total number of cells. Cells were transfected with pLVX-IRES-ZsGreen1-GAS5-free or transduced with GAS5containing virus at a MOI of $40 ; 95 \%$ of cells maintained these plasmids after 3 passages (Fig. 3B).

GAS5 localizes within both the nucleus and cytoplasm. Expression of GAS5 mRNA from wild-type and overexpressed GAS5 SK-Mel-110 cells was determined by FISH and real-time PCR. FISH showed that GAS5 is mainly localized to the cytoplasm in untreated cells but accumulates in the nucleus in GAS5-overexpressed SK-Mel-110 cells (Fig. 4A). The efficiency of the lentivirus transductions was also confirmed by examining GAS5 mRNA levels via real-time PCR. The relative expression of GAS5 mRNA was remarkably increased (45-fold) in GAS5-overexpressing SK-Mel-110 cells, compared 
A
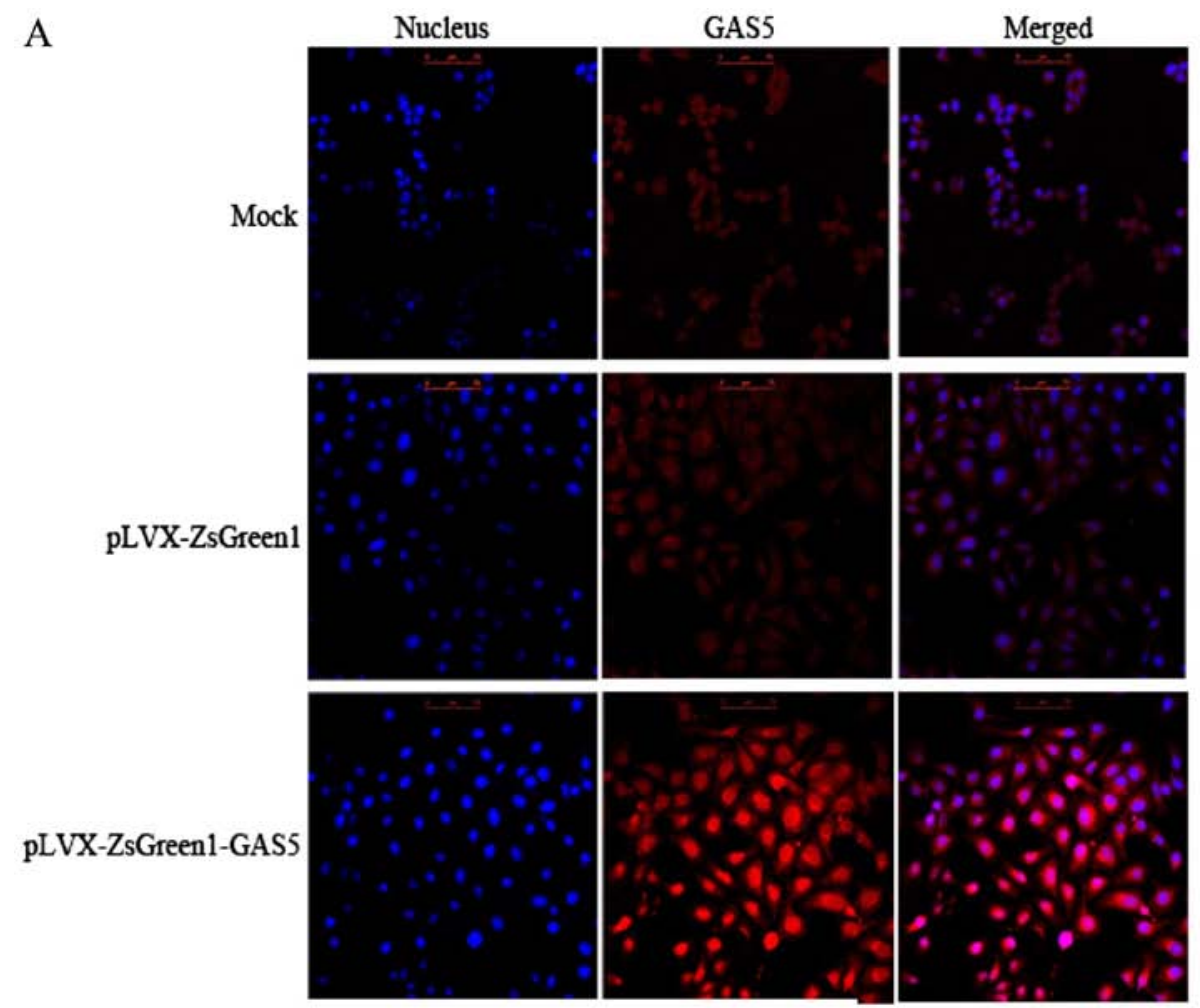

B

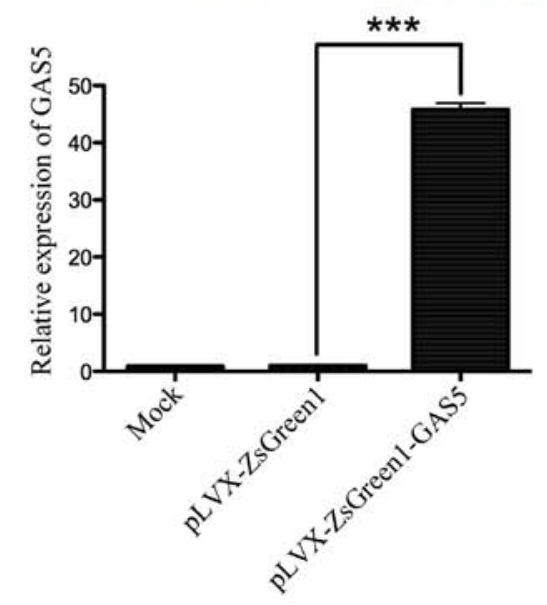

Figure 4. Examining GAS5 expression by RNA FISH and RT-PCR. (A) RNA FISH showed that GAS5 is mainly distributed in the cytoplasm, both in the mock and pLVX-ZsGreen1 SK-Mel-110 cells. A similar quantity of GAS5 mRNA was found in both the nucleus and cytoplasm of pLVX-ZsGreen1-GAS5 SK-Mel-110 cells. Nuclei are stained blue (DAPI) and GAS5 is stained red. (B) RT-PCR was used to quantitatively examine the relative expression of GAS5 in mock, negative control and experimental cells. The results indicate that GAS5 was remarkably increased in pLVX-ZsGreen1-GAS5 SK-Mel-110 cells compared to mock and pLVX-ZsGreen1 SK-Mel-110 cells.

with levels in the negative pLVX-ZsGreen1 and mock control groups (ANOVA; F=1535, $\mathrm{P}<0.01$ ) (Fig. 4B).

GAS5 inhibits the migration and invasion of SK-Mel-110 cells. Both migration and invasion play crucial roles in the metastasis of malignant tumors, which is the main driver behind cancer-related deaths. A classic transwell chamber assay was performed to examine the role of GAS5 in regulating the migratory ability of the melanoma cells. Compared to mock and pLVX-ZsGreen1 cells, pLVX-ZsGreen1-GAS5 cells showed a significant decrease in migratory ability (65.3\%, P<0.001, Fig. 5A). Furthermore, the effects of GAS5 on cell invasion were investigated using a Transwell invasion assay. Three types of cells were cultured in Boyden chambers covered with a Matrigel-coated polycarbonate membrane. Twenty-four hours later, pLVX-ZsGreen1-GAS5 cells migrated through the Matrigel, and a significant decrease in migrated cells was observed in these chambers compared to chambers containing mock and pLVX-ZsGreen1 cells $(55.6 \%, \mathrm{P}<0.001$, Fig. 5B). Taken together, these results indicate that overexpressed GAS5 not only reduces the migration of melanoma cells but also inhibits their ability to invade a matrix, reminiscent of their movement during metastasis.

$M M P 2$ expression levels and activity are reduced in GAS5 overexpressing cells. We examined MMP 2 and MMP13 expression levels using western blot analysis. Overexpressing GAS5 significantly downregulated MMP2 expression by 

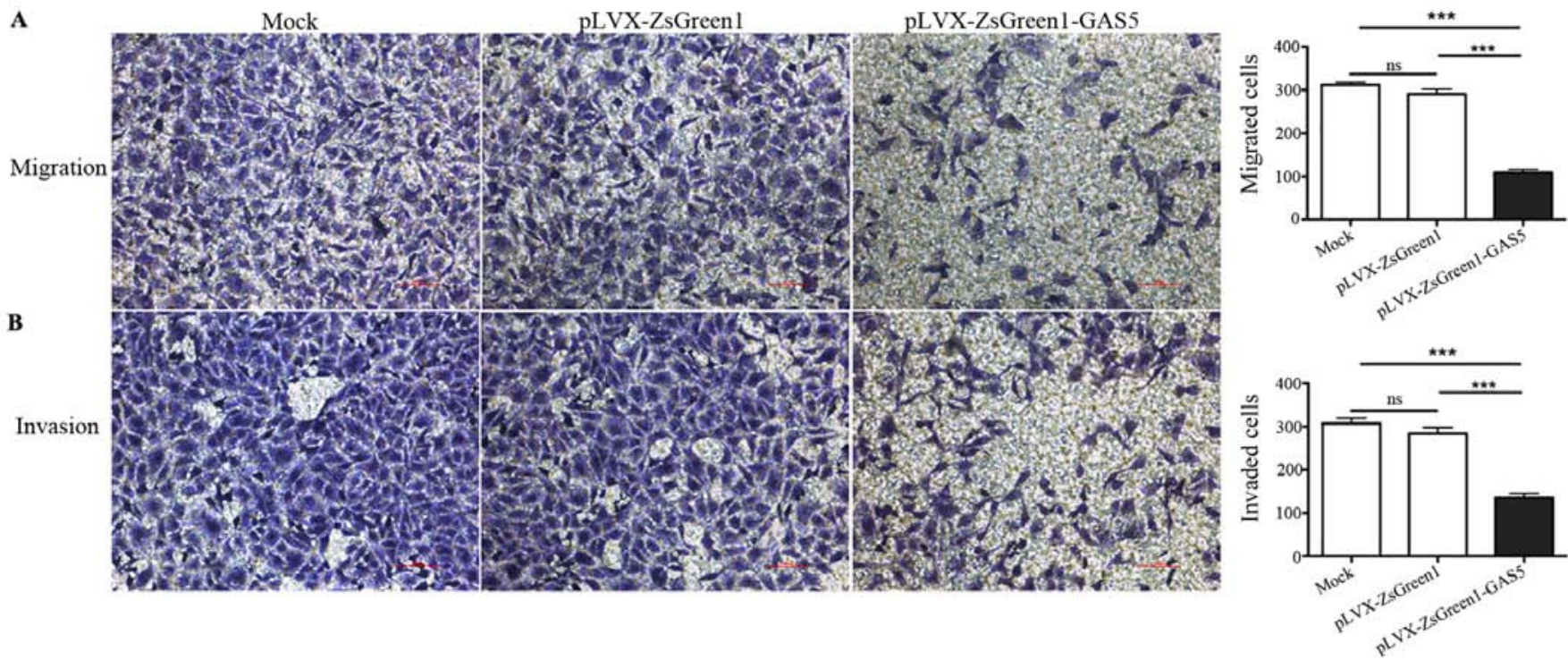

Figure 5. Overexpressing GAS5 reduced the migration and invasion of SK-Mel-110 cells. (A) Upregulating GAS5 expression reduced the ability of SK-Mel-110 cells to migrate in vitro. (B) Overexpressing GAS5 inhibited the invasiveness of SK-Mel-110 cells. Mock, negative control group without transfected cells; pLVX-ZsGreen1, negative GFP control group with cells transfected with ZsGreen1 lentiviral vector alone; pLVX-ZsGreen1-GAS5, experimental group (GAS5 overexpression) with cells transfected with GAS5-ZsGreen1 lentiviral vectors. Data are presented as the mean \pm SD from three independent experiments. Scale bar, $100 \mu \mathrm{m}$.
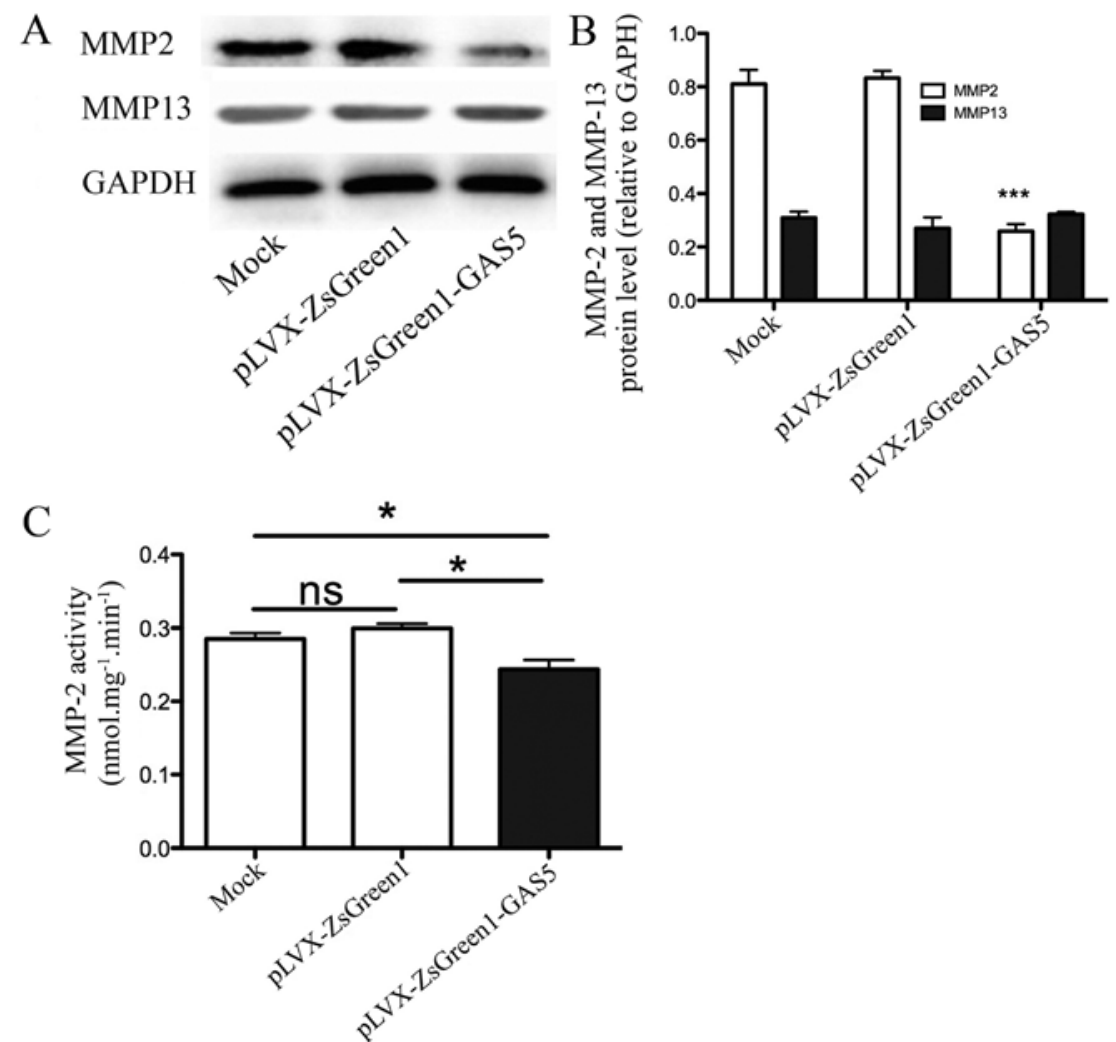

Figure 6. MMP2 protein and activity is downregulated in GAS5-overexpressing SK-Mel-110 cells. Western blot analysis was used to examine the protein expression of MMP2 and MMP13 in three types of melanoma cells (A). Compared to mock, the expression of MMP2 was lower in pLVX-ZsGreen1-GAS5containing cells $(67.9 \%, \mathrm{P}<0.001)$ while MMP13 levels did not change significantly (B). In addition, MMP2 activity decreased by $15.8 \%$ (P<0.05) (C). GAPDH was used as an internal reference. Mock, negative control group without transfected cells; pLVX-ZsGreen1, negative ZsGreen1 control group with cells transfected with ZsGreen1 lentiviral vector alone; pLVX-ZsGreen1-GAS5, experimental group (GAS5 overexpression) with cells transfected with GAS5-ZsGreen1 lentiviral vectors; MMP, matrix metalloproteinase. Data are presented as the mean \pm SD from three independent experiments.

67.9\% ( $\mathrm{P}<0.001)$ (Fig. 6A and B). Given the importance of MMP activity, we also examined how it was affected by GAS5 overexpression. A decrease in MMP2 activity was detected after overexpressing GAS5 (15.8\%, P=0.013) (Fig. 6C), 
consistent with its reduced protein expression. However, there was no significant difference between SK-Mel-110 and SK-Mel-110-GAS5 cells.

\section{Discussion}

The present study aimed to examine the role of lncRNA GAS5 in the regulation of migration and invasion of melanoma cells and to discover the potential underlying mechanisms in this regulation.

We constructed stably expressing GAS5 melanoma cells with which we explored the underlying function of GAS5 in the process of melanoma metastasis. We not only found that GAS5 expression was significantly downregulated in SK-Mel-110 cells compared to HaCaT cells, but we also showed that the overexpression of GAS5 reduced the migration and invasiveness of SK-Mel-110 cells. Further study revealed that overexpressed GAS5 reduced cell invasion, at least partially, by inhibiting MMP2 function. To the best of our knowledge, this is the first study of the relationship between GAS5 and melanoma metastasis.

It has been reported that lncRNAs play important roles in carcinogenesis and the aggressive progression of a spectrum of human cancers $(40,41)$. Some lncRNAs have been implicated in melanoma, such as antisense noncoding RNA in the INK4B locus (ANRIL), BRAF activated non-coding RNA (BANCR), metastasis associated lung adenocarcinoma transcript 1 (MALAT1) and SPRYT-4 intronic transcripts (SPRY4-IT1) (39,42-45). Specifically, chromosomal translocation involving the 1q25 locus, which contains GAS5, has been identified in melanoma (42). Of note, IncRNA GAS5 has gained increasing attention in cancer research because of its ubiquitous downregulation in many cancers (5). However, little is known about its expression in melanoma. Here we show that GAS5 is downregulated in SK-Mel-110 melanoma cells, identified through qRT-PCR and FISH.

It has been demonstrated that lower GAS5 expression is correlated with lymph node metastasis in cervical cancer and hepatocellular carcinoma $(15,21)$. It is thought that low expression of GAS5 is associated with a higher possibility of lymph node metastasis and distant metastasis in non-small cell lung cancer and gastric cancer, although the differences are not significant $(\mathrm{P}=0.652$ and $\mathrm{P}=0.056$, respectively) $(17,19)$. These findings further suggest that lncRNA GAS5 may function as a tumor suppressor and may represent a potential biomarker and new therapeutic target for some cancers. Our results demonstrate that lncRNA GAS5 is downregulated in SK-Mel-110 cells, more so than in $\mathrm{HaCaT}$ cells, and that overexpression of GAS5 inhibits the migration and invasion of these cells.

Migration and invasion are two crucial steps in the process of cancer cell metastasis, which has a high likelihood of leading to a poor prognosis. The invasion of malignant tumors requires the proteolytic degradation of extracellular matrix (ECM) components. Although many proteolytic enzymes and proteases have been implicated in matrix degradation, MMPs are most closely associated with the process of tumor invasion and metastasis (46,47). MMP2, a 72-kDa type IV collagenase also known as gelatinase $\mathrm{A}$, is significantly increased in metastatic melanoma (43\%) compared to normal (5\%) tissue. Compared to patients with negative-to-moderate
MMP2 expression, patients with high MMP2 levels have significantly lower survival rates, and this information could predict patient survival, independent of tumor thickness and ulceration (48). Other studies have shown that higher MMP13 expression, which belongs to the collagenase group, is notably associated with metastasis and poorer survival of melanoma patients (26-28).

Previous studies have provided limited insight regarding the mechanisms by which lncRNA GAS5 inhibits melanoma migration and invasion. We hypothesized that overexpressed GAS5 reduces the invasion of SK-Mel-110 cells by targeting MMP2 and MMP13. In parallel with the reduced cell invasion, a decrease in MMP2 protein expression levels was also observed in these cells, consistent with the predominant role of the 72-kDa gelatinase. MMP2 is an important type IV basal membrane collagenase in human melanoma $(24,49)$. Moreover, both the expression level of MMP2 and its activity, rather than that of MMP13, were negatively associated with the expression of GAS5, which indicates that the lncRNA GAS5 may regulate invasion, at least partially, by modulating MMP2 protein expression levels and activity in SK-Mel-110 cells. MMP2 and MMP13 differ from each other in terms of their amino acid sequences, peptide domain structure and substrate specificity, likely explaining their diverse expression profile in SK-Mel-110 cells (50). Exploring the potential pathway (s) by which GAS5 regulates MMP2 expression and its activity will facilitate the understanding to IncRNA and possibly benefit the prevention and treatment of melanoma. It also provides signposts for identification of targets and predictive biomarkers, as well as potentially useful guidance for decisions about therapy. Therefore, aiming to detect whether there is any direct interaction between GAS5 and MMP2 (both proand active-MMP2), we carried out the RNA pull-down assay. Indeed, there is a protein band close to the $63 \mathrm{kDa}$ marker (active-MMP2 molecule weight is $64 \mathrm{kDa}$ ) yet the mass spectrometry results revealed that this molecule is not MMP2 (data not shown). Given that there is marked accumulation of nucleus GAS5, we suspect that GAS5 may be involved in the transcription of MMP2, our further studies will focus on this potential hypothesis.

Cellular localization plays an important role in understanding the functional roles of lncRNAs, as many lncRNAs regulate nuclear events and thus must localize to the nucleus. Zhang et al discovered that a novel RNA motif, a pentamer sequence of AGCCC with special sequence restrictions at positions -8 and -3 , mediated the nuclear localization of lncRNA BORG, demonstrating that diverse cellular localization influences cell function and biological processes (51). A previous study showed that GAS5 is predominantly located in the cytoplasm of breast cancer cells (52). Similarly, the present study also found that GAS5 locates in the cytoplasm in SK-Mel-110 cells. However, overexpressing GAS5 leads to a noticeable accumulation of GAS5 both in the cytoplasm and nucleus, especially in the nucleus. Considering the previous study that GAS5 can compromise the transcription efficiency of cIAP2 by acting as a 'GRE mimic' in the nucleus, an explanation for its location in the nucleus is that GAS5 exerts its effect on MMP2 possibly by inversely regulating the transcription of MMP2 (52). We hypothesize that accumulation of GAS5 in the nucleus may be one of crucial processes of 
exerting its function. In additon, Renganathan et al revealed that GAS5 is slightly more abundant in the nucleus than that in the cytoplasm in the absence of HhAntag and prevalence in the nucleus was examined after treatment with HhAntag, indicating that HhAntag-induced growth arrest involves accumulation of nuclear GAS5 (18). This study reinforced our hypothesized model. Another potential explanation is that the central dogma of molecular biology holds that 'information' flows from the genes to the structure of the proteins through the formula DNA $\rightarrow$ RNA $\rightarrow$ Protein and exoporting different RNAs from the nucleus to the cytoplasm need various pathways. Large RNAs (rRNA, mRNA, IncRNA and so on) assemble into complicated ribonucleoprotein (RNP) particles and recruit the corresponding exporters via classspecific adaptor proteins (53). Overexpressing GAS5 leads to extremely increased GAS5, resulting in a temporary shortage of the corresponding adaptors, thereby giving rise to the accumulation of nuclear GAS5.

In conclusion, the present study established cell models that overexpressed GAS5 to examine its effects on melanoma cell invasion and migration, as well as other potential functions. Our data solidified an important inhibitive role for GAS5 in the migration and invasion of SK-Mel-110 melanoma cells, which possibly involves MMP2. Our results show that lentivirus-mediated overexpression of lncRNA GAS5 decreases the invasive ability of SK-Mel-110 cells by inhibiting MMP2 expression and activity, but not that of MMP13, possibly at the level of transcription. Our findings provide a new avenue for exploring how lncRNA GAS5 functions in solid tumors. Moreover, these data suggest new drug targets and diagnostic biomarkers for malignant melanoma, and may better inform on the choice of therapies for melanoma patients.

\section{Acknowledgements}

This study was supported by the National Science Foundation of China (nos. 81160246 and 81160421) and the Science and Technology Fund of Yunnan Province (no. 2013FB102). We also appreciate American Journal Experts (AJE) for their language editing service.

\section{References}

1. Siegel RL, Miller KD and Jemal A: Cancer statistics, 2015. CA Cancer J Clin 65: 5-29, 2015.

2. Lo JA and Fisher DE: The melanoma revolution: From UV carcinogenesis to a new era in therapeutics. Science 346: 945-949, 2014.

3. Guil S and Esteller M: RNA-RNA interactions in gene regulation: The coding and noncoding players. Trends Biochem Sci 40 248-256, 2015.

4. Bonasio R and Shiekhattar R: Regulation of transcription by long noncoding RNAs. Annu Rev Genet 48: 433-455, 2014.

5. Pickard MR and Williams GT: Molecular and cellular mechanisms of action of tumour suppressor GAS5 LncRNA. Genes (Basel) 6: 484-499, 2015.

6. Gupta RA, Shah N, Wang KC, Kim J, Horlings HM, Wong DJ, Tsai MC, Hung T, Argani P, Rinn JL, et al: Long non-coding RNA HOTAIR reprograms chromatin state to promote cancer metastasis. Nature 464: 1071-1076, 2010.

7. Mercer TR and Mattick JS: Structure and function of long noncoding RNAs in epigenetic regulation. Nat Struct Mol Biol 20: 300-307, 2013.

8. Mercer TR, Dinger ME and Mattick JS: Long non-coding RNAs: Insights into functions. Nat Rev Genet 10: 155-159, 2009.
9. Smith CM and Steitz JA: Classification of gas5 as a multismall-nucleolar-RNA (snoRNA) host gene and a member of the 5'-terminal oligopyrimidine gene family reveals common features of snoRNA host genes. Mol Cell Biol 18: 6897-6909, 1998.

10. Schneider C, King RM and Philipson L: Genes specifically expressed at growth arrest of mammalian cells. Cell 54: 787-793, 1988.

11. Zhang Z, Zhu Z, Watabe K, Zhang X, Bai C, Xu M, Wu F and Mo YY: Negative regulation of lncRNA GAS5 by miR-21. Cell Death Differ 20: 1558-1568, 2013.

12. Romanuik TL, Wang G, Morozova O, Delaney A, Marra MA and Sadar MD: LNCaP Atlas: Gene expression associated with in vivo progression to castration-recurrent prostate cancer. BMC Med Genomics 3: 43, 2010.

13. Zhang XQ, Sun S, Lam KF, Kiang KM, Pu JK, Ho AS, Lui WM, Fung CF, Wong TS and Leung GK: A long non-coding RNA signature in glioblastoma multiforme predicts survival. Neurobiol Dis 58: 123-131, 2013.

14. Liu Z, Wang W, Jiang J, Bao E, Xu D, Zeng Y, Tao L and Qiu J: Downregulation of GAS5 promotes bladder cancer cell proliferation, partly by regulating CDK6. PLoS One 8: e73991, 2013.

15. Tu ZQ, Li RJ, Mei JZ and Li XH: Down-regulation of long non-coding RNA GAS5 is associated with the prognosis of hepatocellular carcinoma. Int J Clin Exp Pathol 7: 4303-4309, 2014.

16. Lu X, Fang Y, Wang Z, Xie J, Zhan Q, Deng X, Chen H, Jin J, Peng $\mathrm{C}, \mathrm{Li} \mathrm{H}$, et al: Downregulation of gas 5 increases pancreatic cancer cell proliferation by regulating CDK6. Cell Tissue Res 354: 891-896, 2013.

17. Shi X, Sun M, Liu H, Yao Y, Kong R, Chen F and Song Y: A critical role for the long non-coding RNA GAS5 in proliferation and apoptosis in non-small-cell lung cancer. Mol Carcinog 54 (Suppl 1): E1-E12, 2015.

18. Renganathan A, Kresoja-Rakic J, Echeverry N, Ziltener G, Vrugt B, Opitz I, Stahel RA and Felley-Bosco E: GAS5 long noncoding RNA in malignant pleural mesothelioma. Mol Cancer 13: 119,2014

19. Sun M, Jin FY, Xia R, Kong R, Li JH, Xu TP, Liu YW, Zhang EB Liu XH and De W: Decreased expression of long noncoding RNA GAS5 indicates a poor prognosis and promotes cell proliferation in gastric cancer. BMC Cancer 14: 319, 2014.

20. Yin D, He X, Zhang E, Kong R, De W and Zhang Z: Long noncoding RNA GAS5 affects cell proliferation and predicts a poor prognosis in patients with colorectal cancer. Med Oncol 31: 253,2014

21. Cao S, Liu W, Li F, Zhao W and Qin C: Decreased expression of lncRNA GAS5 predicts a poor prognosis in cervical cancer. Int J Clin Exp Pathol 7: 6776-6783, 2014.

22. Jacob A and Prekeris R: The regulation of MMP targeting to invadopodia during cancer metastasis. Front Cell Dev Biol 3: 4, 2015.

23. Shi H, Liu L, Liu L, Geng J, Zhou Y and Chen L: $\beta$-Elemene inhibits the metastasis of B16F10 melanoma cells by downregulation of the expression of uPA, uPAR, MMP-2, and MMP-9. Melanoma Res 24: 99-107, 2014.

24. Toschi E, Rota R, Antonini A, Melillo G and Capogrossi MC: Wild-type $\mathrm{p} 53$ gene transfer inhibits invasion and reduces matrix metalloproteinase-2 levels in p53-mutated human melanoma cells. J Invest Dermatol 114: 1188-1194, 2000.

25. Airola K, Karonen T, Vaalamo M, Lehti K, Lohi J, Kariniemi AL, Keski-Oja J and Saarialho-Kere UK: Expression of collagenases- 1 and -3 and their inhibitors TIMP-1 and -3 correlates with the level of invasion in malignant melanomas. Br J Cancer 80: 733-743, 1999.

26. Zigrino P, Kuhn I, Bäuerle T, Zamek J, Fox JW, Neumann S, Licht A, Schorpp-Kistner M, Angel P and Mauch C: Stromal expression of MMP-13 is required for melanoma invasion and metastasis. J Invest Dermatol 129: 2686-2693, 2009.

27. Corte MD, Gonzalez LO, Corte MG, Quintela I,Pidal I, Bongera M and Vizoso F: Collagenase-3 (MMP-13) expression in cutaneous malignant melanoma. Int J Biol Markers 20: 242-248, 2005.

28. Zhao X, Sun B, Li Y, Liu Y, Zhang D, Wang X, Gu Q, Zhao J, Dong X, Liu Z, et al: Dual effects of collagenase-3 on melanoma: Metastasis promotion and disruption of vasculogenic mimicry. Oncotarget 6: 8890-8899, 2015.

29. Houghton AN, Real FX, Davis LJ, Cordon-Cardo C and Old LJ: Phenotypic heterogeneity of melanoma. Relation to the differentiation program of melanoma cells. J Exp Med 165: 812-829, 1987. 
30. Albino AP, Juan G, Traganos F, Reinhart L, Connolly J, Rose DP and Darzynkiewicz Z: Cell cycle arrest and apoptosis of melanoma cells by docosahexaenoic acid: Association with decreased $\mathrm{pRb}$ phosphorylation. Cancer Res 60: 4139-4145, 2000.

31. Corazzari M, Rapino F, Ciccosanti F, Giglio P, Antonioli M, Conti B, Fimia GM, Lovat PE and Piacentini M: Oncogenic BRAF induces chronic ER stress condition resulting in increased basal autophagy and apoptotic resistance of cutaneous melanoma. Cell Death Differ 22: 946-958, 2015.

32. Tabolacci C, Cordella M, Turcano L, Rossi S, Lentini A, Mariotti S, Nisini R, Sette G, Eramo A, Piredda L, et al: Aloe-emodin exerts a potent anticancer and immunomodulatory activity on BRAF-mutated human melanoma cells. Eur J Pharmacol 762: 283-292, 2015.

33. Li J, Cheng Y, Tai D, Martinka M, Welch DR and Li G: Prognostic significance of BRMS1 expression in human melanoma and its role in tumor angiogenesis. Oncogene 30: 896-906, 2011.

34. Hofacker IL: RNA secondary structure analysis using the Vienna RNA package. Curr Prot Bioinform: Jun 1, 2009 doi: 10.1002/0471250953.bi1202s26.

35. Hofacker IL: Vienna RNA secondary structure server. Nucleic Acids Res 31: 3429-3431, 2003.

36. Du Y, Zhu H, Li D, Wang L, Zhang L, Luo Y, Pan D and Huang M: Lentiviral-mediated overexpression of Akt1 reduces anoxia-reoxygenation injury in cardiomyocytes. Cell Biol Int 38: 488-496, 2014.

37. Li Z, Wang H, Huang S, Zhou L, Wang L, Du C and Wang C: Establishment of stable MRP1 knockdown by lentivirus-delivered shRNA in the mouse testis Sertoli TM4 cell line. Toxicol Mech Methods 25: 81-90, 2015.

38. Li L, Li B, Zhang H, Bai S, Wang Y, Zhao B and Jonas JB: Lentiviral vector-mediated PAX6 overexpression promotes growth and inhibits apoptosis of human retinoblastoma cells. Invest Ophthalmol Vis Sci 52: 8393-8400, 2011.

39. Khaitan D, Dinger ME, Mazar J, Crawford J, Smith MA, Mattick JS and Perera RJ: The melanoma-upregulated long noncoding RNA SPRY4-IT1 modulates apoptosis and invasion. Cancer Res 71: 3852-3862, 2011

40. Gibb EA, Brown CJ and Lam WL: The functional role of long non-coding RNA in human carcinomas. Mol Cancer 10: 38-55, 2011.
41. Spizzo R, Almeida MI, Colombatti A and Calin GA: Long non-coding RNAs and cancer: A new frontier of translational research? Oncogene 31: 4577-4587, 2012.

42. Smedley D, Sidhar S, Birdsall S, Bennett D, Herlyn M, Cooper C and Shipley J: Characterization of chromosome 1 abnormalities in malignant melanomas. Genes Chromosomes Cancer 28: $121-125,2000$.

43. Flockhart RJ, Webster DE, Qu K, Mascarenhas N, Kovalski J, Kretz $\mathrm{M}$ and Khavari PA: BRAFV600E remodels the melanocyte transcriptome and induces BANCR to regulate melanoma cell migration. Genome Res 22: 1006-1014, 2012.

44. Tian Y, Zhang X, Hao Y, Fang Z and He Y: Potential roles of abnormally expressed long noncoding RNA UCA1 and Malat-1 in metastasis of melanoma. Melanoma Res 24: 335-341, 2014.

45. Wapinski $\mathrm{O}$ and Chang HY: Long noncoding RNAs and human disease. Trends Cell Biol 21: 354-361, 2011.

46. Bonnans C, Chou J and Werb Z: Remodelling the extracellular matrix in development and disease. Nat Rev Mol Cell Biol 15: 786-801, 2014.

47. Shay G, Lynch CC and Fingleton B: Moving targets: Emerging roles for MMPs in cancer progression and metastasis. Matrix Biol 44-46: 200-206, 2015.

48. Rotte A, Martinka M and Li G: MMP2 expression is a prognostic marker for primary melanoma patients. Cell Oncol (Dordr) 35: 207-216, 2012.

49. Ray JM and Stetler-Stevenson WG: Gelatinase A activity directly modulates melanoma cell adhesion and spreading. EMBO J 14: 908-917, 1995.

50. Brown GT and Murray GI: Current mechanistic insights into the roles of matrix metalloproteinases in tumour invasion and metastasis. J Pathol 237: 273-281, 2015.

51. Zhang B, Gunawardane L, Niazi F, Jahanbani F, Chen X and Valadkhan S: A novel RNA motif mediates the strict nuclear localization of a long noncoding RNA. Mol Cell Biol 34: 2318-2329, 2014.

52. Mourtada-Maarabouni M, Pickard MR, Hedge VL, Farzaneh F and Williams GT: GAS5, a non-protein-coding RNA, controls apoptosis and is downregulated in breast cancer. Oncogene 28: 195-208, 2009.

53. Köhler A and Hurt E: Exporting RNA from the nucleus to the cytoplasm. Nat Rev Mol Cell Biol 8: 761-773, 2007. 